\title{
How to Determine the Ideal Increased Progesterone Levels Cutoff During Fresh In Vitro Fertilization Cycles: Is it Time to Move Forward-A Short Commentary
}

\author{
Kulvinder Kochar Kaur ${ }^{1 *}$, Gautam Allahbadia ${ }^{2}$ and Mandeep Singh ${ }^{3}$ \\ ${ }^{1}$ Department of Human Reproduction, India \\ ${ }^{2}$ Department of Obstetrics \& Gynecology, India \\ ${ }^{3}$ Department of Neurology, India
}

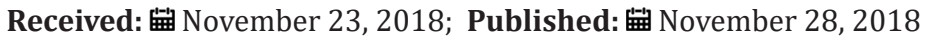

*Corresponding author: Kulvinder Kochar Kaur, Scientific Director, Centre of Human Reproduction, India

\section{Short Communication}

That increased progesterone(P) that is present on the day of human chorionic gonadotropin (HCG)trigger has an effect on the probable chances of a pregnancy resulting following a fresh embryo transfer has been a point that can be debated over the last 25 years [1-3]. A meta-analysis conducted by Venetis et al in 2013 , confirmed the negative effect of progesterone on pregnancy following an ET [3]. They Further confirmed that by using proper analytical approaches one can find this correlation [4]. How this effect is brought about is by changing endometrial receptivity, an approach tested both by basic along with clinical research. Most importantly this support comes from cycles in which increased P do not affect if the embryos are frozen and transferred in subsequent cycle of frozen-thawed embryos [3]. Hence a proposal of freeze all/ freeze only policy was suggested as an answer to manage increased $P$ found by the end of follicular phase.

However, the biggest problem that arises is what is the threshold of increased $\mathrm{P}$ that has to be considered as abnormal and thus needing any intervening procedure. With multiple thresholds getting in the literature more confusion has got created. Hence clinicians are still not sure that on measuring $\mathrm{P}$ on the day of HCG what is the exact threshold which they will employ to decide that they have to cancel a fresh ET and get into freeze-only policy ? This question has been taken up by Hill et al in 2018 [5] by an intense analysis of 7608 ART transfer cycles for getting different $\mathrm{P}$ thresholds and found it varied from $0.4-3.0 \mathrm{ng} / \mathrm{ml}$.Using different methods which kept in mind the sensitivity as well as specificity of every threshold they proposed ,in addition to the cost factor that would result if they used a freeze only(they preferred the term freeze only over freeze all a term adopted by the journal club Global [6]) policy what would the result be. They analyzed that if they used a threshold in between $1.5-2.0 \mathrm{ng} / \mathrm{ml}$, it would prove to be the most cost effective to use the freeze only approach [5]. Although they did find a statistically significant negative effect of $\mathrm{P}$ on pregnancy rates getting found if a threshold of $0.7 \mathrm{ng} / \mathrm{ml}$ although they found if they used this low cut off the number required to treat markedly increased which decreased to 13 with the $1.5-2.0 \mathrm{ng} / \mathrm{ml}$ cutoff.

This analysis is pretty helpful for clinicians in view of their consideration of different variables which they need to keep in mind to make a proper assessment as to when to use the freeze only policy according to the criteria of the P of their clinic. Some limitations of Hills' study as they themselves stressed is potential effectivity of freeze only strategy has never been proved with the use of randomized controlled trials. Thus, what one anticipates the benefits of these strategies, one has not quantified and has only been indirectly calculated based on retrospective data which has been observed. Furthermore, significant factors which influence these like age, total number of oocytes obtained, total number of embryos, their stage of development along with quality of embryos which got transferred. These factors significantly influence the association of $\mathrm{P}$ with live birth rates along with modifying the effect size that is observed [4]. Also, while analyzing no measure of uncertainty like 95\% confidence interval is included and thus exact range of potential outcomes is not considered by Hill et al. Other variables e.g. time of $\mathrm{P}$ measurement, which $\mathrm{P}$ assay is performed, population mix , and what is the effectiveness of various cryopreservation protocols and what is the policy of ET in individual setups which all influence the effect of $\mathrm{P}$ on pregnancy rates and hence change the thresholds accordingly which has been well considered by Hill et al. [5] and thus need to be considered while interpreting the findings.

Significantly however the question lies in the basic reason they carried out this research that is what the optimal threshold regarding increased $P$. One must specify what is abnormal to help make any decision. But trying to divide the population in normal and abnormal just on the base of a single threshold value of a variable that is continuous is a problem and hence landing in situations 
that are counterproductive clinical settings. Like if one decides on the choice of a single threshold as abnormal and then divide into 2 subpopulations, having the similar chance of pregnancy creates problems like say $1.5 \mathrm{ng} / \mathrm{ml}$ is the abnormal average chosen then a patient with a value of $1.45 \mathrm{ng} / \mathrm{ml}$ is supposedly unaffected, while on the contrary a patient having serum $\mathrm{P}$ of $1.52 \mathrm{ng} / \mathrm{ml}$ is fully affected ,using the all or none hypothesis, one expects that there will be a $10 \%$ reduction in the decrease in pregnancy probability in contrast to the unaffected patient, although the difference between the 2 patients is just $0.07 \mathrm{ng} / \mathrm{ml}$ that can be within the margin of error observed in many P assays [3]. More importantly we further consider that the pregnancy rates obtained in these 2 patients is comparable to that of a patient having a level of $0.7 \mathrm{ng} / \mathrm{ml}$ and then further the problem of going on to compare the same with a level of $3.0 \mathrm{ng} / \mathrm{ml}$.

This appears very irrational and further leads to misclassification and then if one tries to dichotomize the statistical power gets decreased on combining with improper adjustment for confound factors that generate type II errors. Senn [7] labeled this issue in biomedical research as "dichotomania". Hence to keep it simple one needs to better utilize the modern statistical techniques, along with continuous data for getting a clearer scenario of the association that is getting investigated.

Thus, it is suggested that need if the hour is one shifts towards increased $\mathrm{P}$ concept to a much more clinically along with statistically sensitive testing of serum P on the day of HCG as a continuous covariate in models that predict live birth following an ET. Importance of this lies in the fact that serum P levels on the day of HCG is the best method that suggests the endometrial receptivity following ovarian stimulation currently. Till date in lieu of methods that are very inefficient, one has not been able to utilize the concept provided by raised serum $\mathrm{P}$, which one can use to maximize the outcomes in assisted reproductive technology. Study by Hill et al. adds further to the knowledge and takes this discussion surrounding increased $\mathrm{P}$ to another level. Still inherent limitations of the ways used currently requires a change in thinking from trying to find what is the most important $\mathrm{P}$ threshold by utilizing the prognostic values which are actually the ones that determine true results by the P levels on the day of HCG.

\section{References}

1. Bosch E, Labarta E, Crespo J, Simon C, Remohi J et al. (2010) Circulating progesterone levels and ongoing pregnancy rates in controlled ovarian stimulation cycles for in vitro fertilization: analysis of over 4000 cycles. Hum Reprod 25(8): 2092-2100.

2. Xu B, Li Z, Zhang H, Jin L, Li Y et al. (2017) Serum progesterone level effects the outcome of in vitro fertilization in patients with different ovarian response: an analysis of more than 10,000 cycles. Fertil Steril 97(6):1321-1327.

3. Venetis CA, Kolibiankis EM, Bosdou JK, Tarlatzis BC (2013) Progesterone elevation and probability of pregnancy after IVF:a systematic review and meta-analysis of over 60,000 cycles. Hum Reprod Update 19(5): 433457.

4. Venetis CA, Kolibiankis EM, Bosdou JK, Lainas GT, Sfontouris IA, et al. (2015) Estimating the net effect of progesterone elevation on day of HCG on live birth rates after IVF: a cohort analysis of 3296 IVF cycles. Hum Reprod 30(3): 684-691.

5. Hill HJ, Healy MW, Richter KS, Parikh T, Devine K, et al. (2018) Defining thresholds for abnormal premature progesterone levels during ovarian stimulation for assisted reproductive technologies. Fertil Steril 110(4): 671-679.

6. Journal Club Global (2017) Is there a progesterone cut off or freeze only?

7. Senn S (2005) Dichotomania: an obsessive-compulsive disorder that is badly affecting the quality of analysis of pharmaceutical trials. Proceedings of the International Statistical Institute, $55^{\text {th }}$ Session, Sydney.

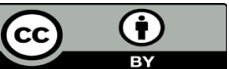

This work is licensed under Creative Commons Attribution 4.0 License

Submission Link: Submit Article

DOI: 10.32474/OAJRSD.2018.02.000127

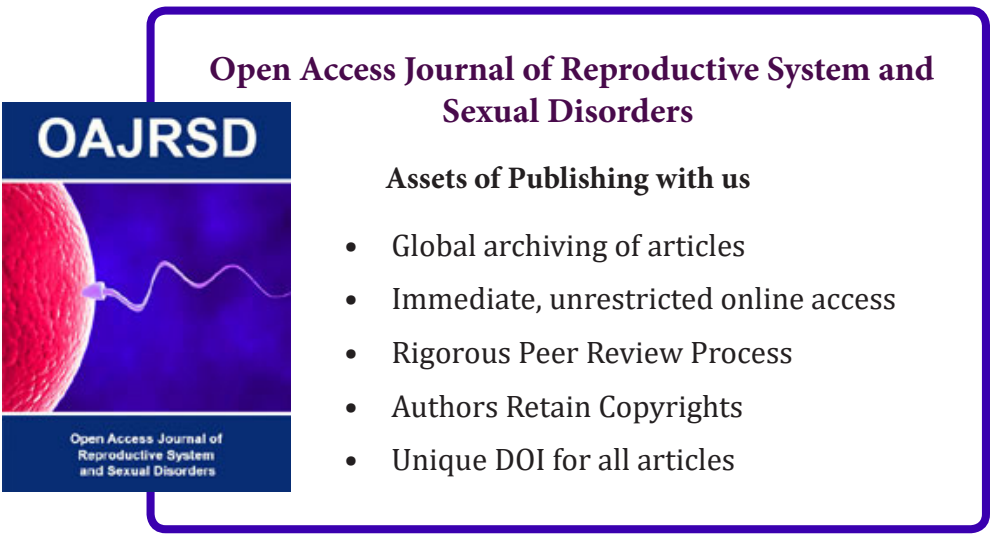

